\title{
CULTURAS NEGOCIADORAS EN MÉXICO E ITALIA: UNA APROXIMACIÓN COMPARATIVA*
}

Recibido: 06 de abril de 2013 - Aprobado: 10 de octubre de 2013

\author{
Manuela Camacho Gómez ${ }^{* *}$
}

\section{RESUMEN}

Este artículo presenta una reflexión comparativa de la forma como negocian los mexicanos y los italianos. El propósito es identificar y analizar las particularidades en la negociación de las culturas mexicana e italiana. Como estrategia metodológica se privilegiaron las indagaciones sobre las percepciones de las personas; el significado, estructura y esencia de una experiencia vivida en algún momento por un individuo, con respecto a una negociación. Los resultados aportan evidencias sobre la forma de negociar relacionadas con filosofía de la negociación, concepción de la contraparte, quiénes negocian, pre negociaciones y apertura de la negociación. . Se evidencia los estilos con que ambas culturas participan en contextos interculturales tanto en asuntos de negocios como personales.

\section{PALABRAS CLAVE}

México, Italia, negociaciones interculturales, administración de negocios internacionales.

\section{CLASIFICACIÓN JEL}

M16

\section{CONTENIDO}

Introducción; 1. Revisión de literatura; 2. Método; 3. México-Italia, las formas de negociar; 4. Análisis comparativo de las variables; 5 . Conclusiones; Bibliografía.

Este artículo presenta resultados del proyecto "Estudio de Relaciones Interculturales como proceso de valor agregado para negocios e instituciones", fue financiado por el Programa de Mejoramiento al Profesorado (PROMEP), México y realizado por el Cuerpo Académico de Estudios Estratégicos para Negocios e Instituciones (CAEENI), Universidad Juárez Autónoma de Tabasco (UJAT), México.

* $\quad$ Licenciada en Relaciones Comerciales por la UJAT, Tabasco, México; maestra en Dirección Internacional por el Instituto Tecnológico Autónomo de México (ITAM), Ciudad de México, México y Doctora en Educación Internacional, Universidad Autónoma de Tamaulipas (UAT), México. Profesora Investigadora de tiempo completo en la División Académica de Ciencias Económico-Administrativas, Universidad Juárez Autónoma de Tabasco (UJAT), México. Avenida universidad s/n, Zona de la Cultura, C. P. 86040, Villahermosa, Tabasco, México. Correo electrónico: manuela.camacho@ujat.mx. 


\section{NEGOTIATION CULTURES IN MEXICO AND ITALY: A COMPARATIVE APPROXIMATION}

\section{ABSTRACT}

This article makes a comparative reflection on the way Mexicans and Italians negotiate. The purpose is to identify and analyze the particularities that Mexican and Italian cultures have in the moment of negotiating. The methodological strategy was focused on the perception of people; the meaning, structure and essence of a lived experience of one individual in a given moment of a negotiation. The results evidences that the different ways of negotiation are related to: Negotiation philosophy, counterpart conception, whom negotiates, pre negotiations and beginning of negotiations. The style of both cultures is evidenced in the way they participate of intercultural contexts both in business as in personal settings.

\section{KEY WORDS}

Mexico, Italy, Intercultural negociations, international business administration.

\section{JEL CLASSIFICATION}

M16

\section{CONTENT}

Introduction; 1. Literary review; 2. Method; 3. Mexico-Italy, negotiation styles; 4. Comparative analysis of variables; 5 . Conclusions; Bibliography.

\section{CULTURAS NEGOCIADORAS EM MÉXICO E ITÁLIA: UMA APROXIMAÇÃO COMPARATIVA}

\section{RESUMO}

Este artigo realiza uma reflexão comparativa da forma como negociam os mexicanos e os italianos. O propósito é identificar e analisar as particularidades na negociação das culturas mexicana e italiana. Como estratégia metodológica se privilegiaram as indagatórias sobre as percepções das pessoas; o significado, estrutura e essência de uma experiência vivida em algum momento por um indivíduo, com respeito a uma negociação. Os resultados contribuem evidências sobre a forma de negociar relacionadas com: filosofia da negociação, concepção da contraparte, quem negociam, pré-negociações e abertura da negociação. . Se evidência os estilos com que ambas culturas participam em contextos interculturais tanto em assuntos de negócios como pessoais.

\section{PALAVRAS CHAVES}

México, Itália, negociações interculturais, administração de negócios internacionais.

\section{CLASSIFICAÇÃO JEL}

M16.

\section{CONTEÚDO}

Introdução; 1. Revisão de literatura; 2. Método; 3. México-Itália, as formas de negociar; 4. Análise comparativa das variáveis; 5 . Conclusões; Bibliografia. 


\section{INTRODUCCIÓN}

Las relaciones interculturales son un tema por demás delicado, pues la cultura es un intangible que determina el comportamiento y forma de actuar de las personas en las diversas naciones. La complejidad cultural abarca desde el comportamiento individual hasta el proceder cotidiano de la sociedad. Es decir, es el estilo de vivir en general, con patrones explícitos o implícitos que son aprendidos y transmitidos simbólicamente y que distinguen a los grupos humanos en un contexto geográfico determinado (Béjar, 2007). En términos de negociaciones, convergen la ambigüedad y la incertidumbre debido a las percepciones de las partes y a la propia forma de negociar de cada uno de ellos. Esto se presenta por el sistema de valores que cada cultura tiene y los modelos de conducta que les han sido inculcados, lo que les lleva a supuestos culturales equívocos o confusos sobre sus interlocutores internacionales (Hendon y Hendon, 1999).

La cultura es un área transversal en los negocios internacionales; los estilos de negociación son influenciadas por esta y por la propia personalidad de quienes llevan a cabo esta actividad. Esa es la razón fundamental por la que se considera que las investigaciones en este tenor permiten disminuir las percepciones distorsionadas y comprender el efecto de las diferencias culturales entre los que negocian, de tal manera que al estudiar las culturas negociadoras se generan conocimientos útiles para ser aplicados en los procesos cotidianos de las empresas e instituciones que están sujetas a negociaciones con pares de otros países y cuyos procesos podrían ser más costosos, tardados o fallidos, cuando sus ejecutivos o representantes tienen desconocimiento de la cultura con la que intentan llegar a acuerdos. Estos estudios hacen uso de teorías probadas sobre las dimensiones culturales propuestas por Hofstede (1982); Trompenaars y Hampden-Turner (1997) y Hall (1989).

Los trabajos previos sobre este tema dan cuenta de una literatura escasa en términos de investigaciones formales respecto a las culturas negociadoras de México e Italia. No obstante, se puede destacar el artículo Chamoun-Nicolas (2005), que propone diez factores: meta, actitud, estilo personal, comunicación, sensibilidad al tiempo, importancia de las emociones, formato de acuerdos, desarrollo de acuerdos, organización de equipos y capacidad de correr riesgos en la negociación.

Asimismo, Díaz-Guerrero (1975), revela que los mexicanos tienen un arraigo a las premisas socioculturales que les han sido impuestas y que en sus relaciones con gente desconocida o poco conocida, se sorprenden porque asumen que la realidad interpersonal puede ser modificada voluntariamente (Díaz-Guerrero, 1975). Es decir, los resultados se enmarcan en la dimensión afectivo-neutral propuesta por Trompenaars y Hampden-Turner. 
Enrique Ogliastri (2001) se guió por 16 variables adaptadas a las dimensiones de Hofstede, Trompenaars y Hampden-Turner y Hall, relativas a distancia de poder, individualismo, incertidumbre, formas de expresión, orientación temporal, emociones, alto o bajo contexto y formalidad del contrato.

Con respecto a la cultura negociadora italiana, se pueden citar trabajos como el de Caterina Cerutti, (2006), donde realiza un análisis comparativo entre las formas de negociar de los alemanes con los italianos, desde una perspectiva de las dimensiones de Hofstede (1982).

Otro trabajo es el de Katz (2007) en el que se presenta un análisis de 50 países entre los que se encuentran México e Italia. Las variables que componen el capítulo de cada país son: interrelaciones, comunicación, contacto inicial, actitudes y estilos, información, estilo predominante de negociación, toma de decisiones y acuerdos y contratos.

Finalmente, está el trabajo de Camacho (2010) donde se analiza el estilo de negociación de la cultura italiana a partir de las variables propuestas por Ogliastri (1999).

En consecuencia, se estima que esta investigación es conveniente y relevante para las organizaciones, ya que contribuye a la generación de conocimiento en materia de negociaciones interculturales en América Latina y su relación con pares de países de Europa.

Por lo tanto, identificar y analizar las particularidades negociadoras de las culturas mexicana e italiana es congruente con las preguntas de investigación que se plantearon para indagar cómo negocian los mexicanos (e italianos), porqué negocian de esa manera, cuál es su proceso característico, y qué recomendaciones se pueden hacer a ellos y a quienes negocien con ellos (Ogliastri, 1998).

El diseño de la investigación cualitativa con enfoque fenomenológico permite conocer las percepciones de las personas; el significado, estructura y esencia de una experiencia vivida en negociaciones con otras culturas, por lo que el análisis de resultados proporciona una ilustración sobre sus apreciaciones y alcances con el objeto de entender y plantear prescripciones básicas para negociar con estas personas.

Así, el artículo muestra algunas características de negociación mexicana e italiana, basadas en 45 y 23 entrevistas, respectivamente; las cuales fueron aplicadas a ciudadanos de diversas nacionalidades, entre los que se encuentran mexicanos, colombianos, chilenos, venezolanos, salvadoreños, nicaragüenses y bolivianos, entre otros. Sin embargo, los perfiles de las culturas negociadoras que se plantean 
en este artículo son solo aproximaciones; no se puede afirmar categóricamente que los resultados de la investigación representen la generalidad de cómo negocian las personas de ambos países, sino más bien, dan pauta a la puesta en marcha de otras investigaciones sobre culturas negociadoras en América Latina y estudios comparativos entre estas y las de otras regiones del mundo como Europa y Asia que son las menos abordadas.

\section{REVISIÓN DE LITERATURA}

\subsection{Cultura}

La cultura es por sí misma, una proveedora de renovación, innovación, invención y creatividad. Es por ello que la diversidad cultural influye e impulsa las buenas relaciones entre países y entre personas. En el caso de las negociaciones es fundamental tomar en cuenta, respetar y conocer la pluralidad cultural de las contrapartes (UNESCO, 2001). Se trata de una interacción social que presupone caminos comunes para procesar información entre las personas que interactúan entre sí. Esto tiene impacto en la forma de hacer negocios, particularmente cuando participan en contextos internacionales. La dependencia mutua de los actores se presenta porque los hechos que juntos construyen, están acoplados a un sistema de entendimiento, es decir, se trata de definir el modo en que se ha de abordar una situación por un grupo determinado (Trompenaars y Hampden-Turner, 1997).

En los contextos culturales, los procesos de negociación se ven influenciados por los patrones de pensamiento, sentimientos, actitudes, costumbres, creencias y valores culturales propios de la naturaleza humana. Aunque también debe tomarse en cuenta que muchas veces coexisten varias culturas dentro de la misma nación, derivado del contexto de las mismas (Horst y Colonel, 2007; Ogliastri, 1998). Por ejemplo en México, los negociadores del Distrito Federal tienden a ser más competitivos, pero también más preparados y exigentes con sus contrapartes, esto se debe a la ubicación geográfica, el dinamismo y el tamaño de sus empresas; en tanto que los del sureste del país, son más cálidos, menos proclives a los riesgos y se orientan al largo plazo, lo que hace más lento el proceso.

Por lo tanto, la cultura se erige como una parte central del proceso de negociar, ya que en el ambiente global hace posible la comprensión de las características propias y las de otros, así como la factibilidad de interactuar con diferentes grupos sociales en todo el mundo. En este sentido, las empresas deben desarrollar una perspectiva multicultural para que sus negociadores posean el conocimiento cultural pertinente y suficiente para participar en su despliegue de intercambios hasta 
encontrar puntos de acuerdo, y resolver convergencias que ayuden al logro de los propósitos de cada parte (Rosen et al., 2000).

Cuando las divergencias culturales no se aprecian como un área de oportunidad, hacen más compleja la identificación de los puntos comunes y las discrepancias tienden a incrementarse y a obstaculizar el alcance de óptimos resultados para quienes negocian. Esto ocurre porque el contexto socio-político, económico, legal y tecnológico determina las ventajas y desventajas asociadas con la actividad empresarial, por lo que es necesario que los negociadores también tengan acceso a un entrenamiento transcultural que les permita el alcance pleno de sus propósitos de negociación (Martínez, Corredor y Herazo, 2006).

Las diferencias culturales afectan el contexto empresarial; por lo tanto la cultura es el camino a través del cual las personas resuelven problemas y reconcilian dilemas cuando estos se presentan, lo que conduce a plantear acciones para desaparecerlos (Trompenaars and Hampden-Turner, 1997).

Es evidente que la cultura, por su magnitud, ha sido motivo de estudio por diversos investigadores. En términos organizacionales fue Geerth Hofstede quien se enfocó a realizar investigaciones en 70 países, con el objeto de conocer la influencia de la cultura en los valores que poseen las personas en sus actividades laborales. De tal manera que la participación transnacional de las organizaciones conviene estar basada en una cultura propia. Sus hallazgos se concentraron en un principio en cuatro dimensiones: distancia de poder, individualismo, masculinidad y evasión a la incertidumbre y fueron publicados en 1980 en el libro titulado Culture's Consequences. Posteriormente, se llevaron a cabo estudios complementarios para agregar la quinta y sexta dimensión (Hofstede Centre s/f) (Ver tabla 1).

Tabla 1. Dimensiones culturales de Hofstede

\begin{tabular}{|l|l|}
\hline \multicolumn{1}{|c|}{ Dimensión } & \multicolumn{1}{c|}{ Alcance } \\
\hline Distancia de poder & $\begin{array}{l}\text { Se refiere al uso del poder en función de la jerarquía organiza- } \\
\text { cional. Se asume la presencia del componente de desigualdad. }\end{array}$ \\
\hline Individualismo & $\begin{array}{l}\text { Compete al grado de integración que la persona tiene o no en un } \\
\text { grupo determinado. Aquí se considera la contraparte colectividad. }\end{array}$ \\
\hline Masculinidad & $\begin{array}{l}\text { Relacionada con los valores y actitudes según el género. En esta } \\
\text { dimensión, existe el complemento femineidad. }\end{array}$ \\
\hline Incertidumbre & $\begin{array}{l}\text { Indica el grado en que los integrantes de una cultura se sienten } \\
\text { cómodos o incómodos en situaciones no estructuradas que } \\
\text { causan incertidumbre. Se asume que las culturas tienden a evadir } \\
\text { o a aceptarla. }\end{array}$ \\
\hline
\end{tabular}


Culturas negociadoras en México e Italia: una aproximación comparativa

\begin{tabular}{|l|l|}
\hline \multicolumn{1}{|c|}{ Dimensión } & \multicolumn{1}{c|}{ Alcance } \\
\hline Largo plazo & $\begin{array}{l}\text { Es una dimensión que considera la capacidad de ahorro y per- } \\
\text { severancia. La contraparte es la orientación a corto plazo que se } \\
\text { asocia con obligaciones sociales y prestigio personal }\end{array}$ \\
\hline Formas de expresión & $\begin{array}{l}\text { Concerniente a la forma en que una sociedad da la libertad de } \\
\text { manifestar impulsos o expresiones de la naturaleza humana, o la } \\
\text { restricción de las mismas, a través de normas sociales estrictas } \\
\text { y expresiones controladas. }\end{array}$ \\
\hline
\end{tabular}

Fuente: Elaboración propia con información de Hofstede (2011); Hofstede Centre y Hofstede site

Por su parte, Fons Trompenaars y Charles Hampden-Turner publicaron en 1997 la obra Riding of Waves of Culture, en la cual se proponen y analizan siete componentes sobre las diferencias culturales, a saber: universalismo contra particularismo; individualismo contra comunidad; afectividad contra neutralidad, especificidad contra difuso; logro o adscripción; ubicación del control (interno o externo); y orientación temporal (ver tabla 2).

Tabla 2. Diferencias culturales de los países

\begin{tabular}{|l|l|}
\hline $\begin{array}{l}\text { Universalismo-particu- } \\
\text { larismo }\end{array}$ & $\begin{array}{l}\text { Describe sobre si la cultura se enfoca más a las relaciones o a } \\
\text { los aspectos legales, para validar acuerdos formales. }\end{array}$ \\
\hline $\begin{array}{l}\text { Individualismo-comu- } \\
\text { nitario }\end{array}$ & $\begin{array}{l}\text { Lo refiere hacia preferencia de actuar y decidir de modo personal } \\
\text { o comunitario. }\end{array}$ \\
\hline Afectivo-neutral & $\begin{array}{l}\text { Concierne a la expresión de sentimientos, emociones y comuni- } \\
\text { cación que influye en el contacto con otras personas }\end{array}$ \\
\hline Específico-difuso & $\begin{array}{l}\text { Explica el grado de involucramiento y la manera de expresarse } \\
\text { de la gente en sus interrelaciones, las cuales pueden ser fluidas } \\
\text { o lentas }\end{array}$ \\
\hline Logro o adscripción & $\begin{array}{l}\text { Es el estatus que las personas tienen por sí mismas o a través } \\
\text { de otros. }\end{array}$ \\
\hline Control & $\begin{array}{l}\text { Está enfocado a la creencia cultural de poseer un control (interno } \\
\text { o externo) que puede tener alcance en su contexto. }\end{array}$ \\
\hline Orientación temporal & $\begin{array}{l}\text { Está relacionado con las inclinaciones hacia experiencias pasadas } \\
\text { para atender situaciones presentes, o bien, invertir en el presente } \\
\text { para el futuro. También analiza el uso del tiempo, bien sincrónico } \\
\text { o asincrónico. }\end{array}$ \\
\hline
\end{tabular}

Fuente: elaboración propia con información de Trompenaars y Hampden-Turner (2007).

Otra dimensión a considerarse en la cultura es el grado y formas de comunicación y su influencia en la flexibilidad y formalidad de las negociaciones. De esta 
manera Hall (1989), citado por García (2005), propone el análisis de las culturas de alto contexto (CAC), referidas a aquellas donde la relevancia del contexto es más fuerte que a la comunicación hablada; es decir, la palabra no es concluyente y se tiende a recurrir menos a documentos legales; lo que provoca la lentitud en las negociaciones. En estas culturas, la posición social y el estatus son de alta relevancia. Los negocios tienden a requerir de más tiempo, ya que se necesita establecer una relación personal que genere confianza entre las partes. En cuanto a la cultura de bajo contexto $(\mathrm{CBC})$, los argumentos son claros y transmiten la mayor parte de la información que las partes precisan. Los documentos legales se consideran indispensables. Con estas condiciones, los negocios son más ágiles debido a que los detalles se analizan en menor tiempo.

Bajo estas premisas corresponde a los directivos y funcionarios desarrollar competencias interculturales relacionadas con: valores, estilo de pensamiento, conocimientos, habilidades, actitudes, experiencia. Además, es de relevancia desarrollar valores relacionados con la honestidad, el respeto hacia el mundo que nos rodea, la franqueza y la sinceridad, y consideraciones especiales a favor de las partes más vulnerables. En este sentido, es preciso poseer un estilo de pensamiento flexible, propositivo, no-categorizador, holístico y hábil para comprender otras formas de pensar, además de poseer competencias comunicativas (Pariente, 2003).

En las negociaciones, cada país tiene en sus procesos de negociación un componente cultural que los distingue y que determina los estilos de negociar de sus representantes. Es el factor que más impacta en la comprensión y expectativas de las partes que participan en la búsqueda de un acuerdo negociado, por lo es importante que las partes comprendan las implicaciones de un proceso intercultural, sus ventajas y desventajas y la preparación que tal hecho exige. En las culturas negociadoras se aprecian diferencias relacionadas con el uso del tiempo, la sensibilidad de lo personal, la percepción de lo específico y lo general. Es decir, más allá de las nacionalidades, existen diferencias culturales entre los hombres y las mujeres (Chamoun-Nicolás, 2007).

\subsection{Los países}

\subsubsection{Estados Unidos Mexicanos}

Hasta el año 2010, la república mexicana contaba con 112322757 millones de habitantes (INEGI, 2010). Este país se distingue por sus contrastes y convergencias culturales; hay personas que señalan que negociar en México es hacerlo con varias culturas a la vez. Tal afirmación obedece a que el estilo de negociación cambia según la ubicación geográfica donde se lleve a cabo el proceso negociador. Sin embargo, 
los mexicanos sobresalen por su cordialidad, alegría y deseos de aprender de otros.

La geografía mexicana posee colindancia fronteriza, al norte, con los Estados Unidos de América, al sur, con Guatemala y Belice. Su clima va desde cálido húmedo (5 \%) hasta muy seco (21 \% de su superficie) (OEI, s. f.). Su proximidad con los Estados Unidos de América es una ventaja que ha favorecido especialmente al ambiente de los negocios.

En materia económica, la competitividad de sus sectores es diferente, según sea la región (OEI, s.f.). Dentro de sus sectores estratégicos se encuentran el industrial, donde destacan la automotriz, autopartes, aeronáutica, siderúrgica, electrónica, alimentos, bebidas, tabaco, textil, confección, cuero, calzado, farmacéutico, químico y tecnologías de información; y los servicios como la construcción y el turismo, que juntos generan más del 90 \% del PIB nacional (INECC, 2009; Secretaría de Economía s/f).

El mexicano tiende a compararse, lo que le lleva a encontrar diferencias y similitudes; por lo tanto, robustece los aspectos positivos y negativos entre ellos y la cultura con la que se comparan. Tal actitud puede generar el complejo de inferioridad, ya que no se acepta como es (Magallón, 2007). En general, la gente de México está muy arraigada a sus costumbres y a su cultura, hechos que se traducen en calidez y hospitalidad en su interrelación personal. En materia de negociaciones, las tendencias actuales dan muestra de un mexicano preparado, conocedor de lo que negocia, educado, dispuesto a contribuir a los logros de su contraparte, buen anfitrión y con un enfoque de multi y transculturalidad.

\subsubsection{República Italiana}

La república italiana es miembro fundador de la Unión Europea (UE). Hasta 2010, Italia contaba con un total de 59364690 millones de habitantes. La población femenina es mayoría y representa el $51.41 \%$ de la población (datos macro s/f). Se destaca por contar con una geografía muy variada que se explaya desde los Alpes hasta el centro del Mediterráneo. Es limítrofe con Francia, Suiza, Austria y Eslovenia y de acuerdo con las estadísticas de la UE, es el tercer país de ese grupo que recibe mayor número de turistas al año. Acoge a dos Estados independientes: la Ciudad del Vaticano, en Roma, y la República de San Marino (CAMCIG, s.f.).

La economía italiana posee áreas de oportunidad en sectores como la siderurgia y construcción naval, y el textil. Los principales recursos minerales son carbón, mercurio, zinc, potasio, mármol, barita, asbestos, fluorita, gas natural y reservas de petróleo crudo. Posee grandes ventajas en los sectores alimenticio, confección, vidrio, metalurgia, automovilístico, maquinaria agrícola, material ferroviario, 
construcciones aeronáuticas y refinamiento de fibras sintéticas, por citar las más significativas. Asimismo, en el sector terciario, que representa el $63 \%$ del personal ocupado, el turismo es uno de los principales generadores de empleo y de divisas (SGM, s/f; Ambitalia, s. f.).

En este país europeo también cada región tiene su cultura y formas de hacer negocios, por lo que es imprescindible adaptarse a las particularidades de cada zona. En general los italianos son muy emprendedores y tienen inclinación por salir de su país. Se estima que aproximadamente 100 millones de italianos habitan fuera de su nación. En cuanto a las negociaciones los italianos prefieren establecer relaciones con personas conocidas o que son presentadas por otros que son de confianza, lo que es conocido como raccomendazione (Instituto de Fomento Región de Murcia, 2012). El italiano, en general, es una persona educada, en exceso emotiva, con expresiones gesticulares distintivas, orgullosos de su país y con un marcado arraigo a sus raíces culturales.

\section{MÉTODO}

El método utilizado fue el cualitativo y el diseño de la investigación siguió un enfoque fenomenológico que apunta hacia las experiencias individuales subjetivas de los entrevistados. Se pretendía conocer las percepciones de las personas y el significado, estructura y esencia de una experiencia vivida en algún momento, por un individuo, grupo o comunidad respecto de un fenómeno (Hernández, Fernández, Baptista, 2010).

El objetivo de la investigación fue identificar y analizar las particularidades negociadoras de las culturas mexicana e italiana, a través de indagaciones precisas, para ofrecer una explicación sobre sus apreciaciones y alcances con el objeto de entender y plantear prescripciones básicas para negociar con estas personas.

En congruencia con el objetivo del estudio, las preguntas de investigación se orientaron a indagar cómo negocian los mexicanos (e italianos), porqué negocian de esa manera, cuál es su proceso característico, y qué recomendaciones se pueden hacer a ellos y a quienes negocien con ellos (Ogliastri, 1998). Se trata por tanto, de una descripción cultural basada en la teoría de negociación y se estima que esta investigación es conveniente y relevante socialmente y contribuye a la generación de conocimiento en materia de negociaciones interculturales en América Latina y su relación con pares de países de Europa.

Después de analizar los aportes y publicaciones y colaborar, por un periodo corto, con Ogliastri, su metodología para investigar las culturas negociadoras fue suficientemente atractiva y convincente para replicarla, en este caso, con mexicanos 
e italianos. Se eligió esta metodología porque se buscaba comprender el fenómeno de la negociación desde la perspectiva comparativa de dos culturas negociadoras, a través de la exploración de experiencias, opiniones, y en general, cómo los mexicanos e italianos perciben subjetivamente su realidad, en un ambiente natural y en relación con su contexto (Hernández, Fernández, Baptista, 2010).

Así, lo que marcó la pauta para realizar la investigación con mexicanos e italianos, fue impartir la cátedra de negociaciones internacionales durante 13 años en una escuela de negocios en Villahermosa, Tabasco, México, motivo suficiente para adquirir el compromiso de añadir más conocimiento sobre las culturas negociadoras y, lógicamente, el interés prioritario fue estudiar a los mexicanos en sus procesos de negociación, pero a través de las variables propuestas y probadas en múltiples ocasiones por Ogliastri (1999).

Por el tipo de estudio a realizar y aprovechando el contexto, la población elegida para el caso de la cultura negociadora mexicana, fueron los estudiantes de la Maestría en Administración y directivos de empresas locales y nacionales. Es importante mencionar que la mayoría de los estudiantes del posgrado de referencia, son al mismo tiempo, directivos de empresas privadas, nacionales e internacionales; funcionarios de empresas paraestatales; funcionarios de gobierno y consultores de negocios. Las nacionalidades, aunque predominantemente mexicanas, también incluyeron alumnos provenientes de Colombia, Chile, Venezuela y mexicanos que habían vivido en otros países como Estados Unidos, Canadá y España.

Con respecto a Italia, la población también estuvo compuesta por estudiantes de la Maestría en Administración del INCAE Business School de Managua, Nicaragua, originarios de diversas nacionalidades, especialmente de Centro y Sudamérica. Muchos de ellos con vivencias profesionales en negociaciones en sus países de procedencia y con colegas de diversas partes del mundo.

La muestra se determinó a partir del contexto que guardan las negociaciones internacionales con énfasis en la cultura de quienes negocian, y por su enfoque cualitativo no representa a una población, ya que desde la perspectiva fenomenológica, el objetivo fue analizar valores, protocolos y significados de las personas que habían negociado con mexicanos o italianos; se decidió trabajar con una muestra de casos tipo, donde el fin fue la riqueza, profundidad y calidad de la información, no la cantidad ni la estandarización de las mismas (Hernández, Fernández, Baptista, 2010). El número de casos (tamaño de la muestra) fue intencional o dirigido, ya que no se tenía la certeza del número de personas que habrían negociado precisamente con las culturas que se estudiaban. Por el tipo de fenómeno a analizar, el número de entrevistas se cerró cuando se estimó haber llegado a la saturación de categorías. 
El instrumento utilizado fue la entrevista, desarrollado y probado por Ogliastri en diversas investigaciones sobre culturas negociadoras latinoamericanas y su interrelación con japoneses, angloamericanos, franceses y del Medio Oriente. Asimismo, el estudio de las negociaciones de colombianos, venezolanos, japoneses, franceses, guatemaltecos, peruanos, israelitas, Medio Oriente, además de las investigaciones comparativas entre México y Argentina; Colombia y Holanda; Colombia y Venezuela; Colombia y Japón, y alemanes con latinoamericanos. Todo esto en un período amplio de 1987 a 2010 que avala sus publicaciones en diversos países (Ogliastri, página personal).

Para la recolección de la información en campo, en el caso de los mexicanos el acopio fue realizado por la autora, estudiantes de posgrado y asistentes de investigación. Las entrevistas para conocer la cultura italiana se llevaron a cabo por los asistentes de Ogliastri y por la autora.

Por tratarse de una investigación cualitativa, el análisis de los datos se realizó en paralelo con la recolección, debido a que este no fue estandarizado, y a que los datos fueron variados en su amplitud y profundidad. En este orden de ideas se organizaron y codificaron los hallazgos, generando significados y categorizando temas y relaciones entre conceptos y las variables cualitativas estudiadas, en términos de procesos de negociación que seguían ambas culturas. En consecuencia, el estilo de negociación que los entrevistados siguieron y manifestaron, arrojó información sobre percepciones, imágenes mentales, interacciones, pensamientos, vivencias en el lenguaje de los participantes de manera individual. En general, la experiencia negociadora que ellos decidieran compartir (Hernández, Fernández, Baptista, 2010). Por esta razón se procuró entender los motivos subyacentes, los significados y razones internas del comportamiento de los entrevistados, teniendo presente la literatura revisada.

Es importante mencionar que los trabajos de recolección se llevaron a cabo en períodos diferentes: 2011-2013 para mexicanos y 2004-2006 para italianos. Las entrevistas fueron escritas y grabadas, y se transcribieron textualmente. La descripción fue realizada con procedimientos manuales de análisis informativo.

Referente a los criterios de confiabilidad y validez del estudio, estas se determinaron por el uso de bitácora de análisis donde se hicieron ajustes de codificación, anotaciones respecto a categorías y supuestos que fueron surgiendo durante el proceso, además de las reflexiones analíticas del investigador. En términos cualitativos la confiabilidad y validez se traducen en credibilidad y confirmación que consiste en la capacidad de comunicar el lenguaje, pensamientos, emociones y puntos de 
vista de los entrevistados (Hernández, Fernández, Baptista, 2010). La validez en las investigaciones cualitativas por tanto, se fundamenta en el modo de recoger los datos, de captar cada evento desde sus diferentes puntos de vista, de vivir la realidad estudiada y de analizarla e interpretarla en su contexto. Con ello se supera la subjetividad y se añade rigor y seguridad a las conclusiones (Martínez, 2006).

Para fines de este trabajo, se seleccionaron cinco variables, con las cuales se realizó la aproximación comparativa de las dos culturas referidas.

\section{MÉXICO-ITALIA, LAS FORMAS DE NEGOCIAR}

En este artículo se analizan las variables 1, 2, 6, 10 y 11; las cuales ilustran la forma de negociar de las culturas mexicana e italiana, con el propósito de dar a conocer la manera como la generalidad de las personas procedentes de México o Italia conciben o actúan en ese conjunto de variables (Ver tabla 3).

Tabla 3. Variables de negociación analizadas México-Italia

\begin{tabular}{|c|c|c|c|}
\hline Núm. & Variable & \multicolumn{2}{|c|}{ País } \\
\hline & & México & Italia \\
\hline 1 & $\begin{array}{l}\text { Filosofía de } \\
\text { negociación }\end{array}$ & $\begin{array}{l}\text { Distribución de valor con ten- } \\
\text { dencia a negociaciones inte- } \\
\text { gradoras y gran interés por el } \\
\text { largo plazo. }\end{array}$ & $\begin{array}{l}\text { Distribución de valor y tenden- } \\
\text { cia al largo plazo. }\end{array}$ \\
\hline 2 & $\begin{array}{l}\text { Concepción de } \\
\text { la contraparte }\end{array}$ & $\begin{array}{l}\text { Trato cordial y afectuoso, de } \\
\text { manera especial cuando existen } \\
\text { lazos de amistad. Muestran } \\
\text { despliegues de amabilidad y } \\
\text { cordialidad, manifiestan interés } \\
\text { por las buenas relaciones. }\end{array}$ & $\begin{array}{l}\text { Trato impersonal, salvo cuando } \\
\text { existe un nivel de confianza sufi- } \\
\text { ciente, por lo general el italiano } \\
\text { se muestra con mucha educa- } \\
\text { ción y trata de ser agradable }\end{array}$ \\
\hline 6 & $\begin{array}{l}\text { Quiénes nego- } \\
\text { cian }\end{array}$ & $\begin{array}{l}\text { Negocia el de más alta jerarquía; } \\
\text { la decisión de quién negocia } \\
\text { está en función del tamaño de } \\
\text { la empresa representada. El que } \\
\text { negocia posee una mezcla de } \\
\text { jerarquía-conocimiento. }\end{array}$ & $\begin{array}{l}\text { Negocia quien conoce sobre } \\
\text { los asuntos y forma de abordar } \\
\text { la negociación. Cuando el con- } \\
\text { flicto no se soluciona por los } \\
\text { negociadores asignados, entra } \\
\text { alguien de mayor jerarquía. }\end{array}$ \\
\hline 10 & $\begin{array}{l}\text { Pre negocia- } \\
\text { ciones (pre- } \\
\text { paración de la } \\
\text { negociación) }\end{array}$ & $\begin{array}{l}\text { Se preparan antes de iniciar } \\
\text { una negociación formal, buscan } \\
\text { relacionarse e investigar a su } \\
\text { contraparte, prefieren eviden- } \\
\text { cias de lo que va ofrecer y lo que } \\
\text { ofrecerá su contraparte. }\end{array}$ & $\begin{array}{l}\text { Utilizan un esquema de in- } \\
\text { tercambio de información, a } \\
\text { manera de consensos y de } \\
\text { acuerdos previos. }\end{array}$ \\
\hline
\end{tabular}




\begin{tabular}{|l|l|l|l|}
\hline 11 & Aperturas basadas en criterios & Apertura con posiciones con- \\
objetivos relacionados con & cretas, muy cercanas al punto \\
asuntos a negociar; con ten - & de resistencia. Se proveen de \\
dencia al regateo. Se adaptan & información suficiente. Apego \\
a las culturas no regateadoras. & a las normas y leyes, principal \\
Suelen proveerse de informa & argumento en la negociación y \\
& ción estratégica y estudiar a su & la base de los cierres. \\
& contraparte. & \\
\hline
\end{tabular}

Fuente: elaboración propia, con información de Camacho (2010).

\section{ANÁLISIS COMPARATIVO DE LAS VARIABLES}

\subsection{Filosofía de negociación (¿Cómo negocian los mexicanos y los italianos?)}

La filosofía de negociación mexicana se destaca por llevar a cabo un proceso de distribución de valor, con posiciones duras e inflexibles al principio, de forma primordial cuando no tienen confianza con la contraparte; su forma de expresión es policrónica y en ocasiones ambigua, sin comprometerse demasiado. Los mexicanos prefieren no asumir riesgos; sin embargo, confían en la contraparte. Me gustó la flexibilidad en las negociaciones dentro de los marcos de gobernación, apertura y estabilidad política. También me agrada la seriedad en la negociación, que se apliquen las regulaciones gubernamentales, así como las normatividades del medio ambiente, que quede un legado para la comunidad. Si Los países respetan eso, eso da continuidad en el negocio. Que se cumpla como debe de ser, la imagen corporativa se consolida (9).

En cuanto al manejo de las emociones, suelen ser fraternos, hospitalarios y tienden hacia la búsqueda de amistad para sentirse más cómodos y presumir sus buenas relaciones. Sus argumentos y posiciones en las negociaciones profesionales están determinados por el análisis e investigación que realizan con antelación sobre la contraparte y sus propias posiciones. Su perspectiva temporal de acuerdo a las entrevistas realizadas denota una gran inclinación en las relaciones de largo plazo. "... el mexicano suele ser muy emocional empiezan con una risa, una carcajada y suelen terminar con algún mal chiste" (13).

Que los (mexicanos) que vayan a negociar fuera del país siempre tengan un mismo estilo de negociación, que la misión y visión de esta negociación sea única, corporativa que no admita clasificaciones. La negociación es única es como tu huella digital, y las variantes son las estrategias que si pueden cambiar de acuerdo a los países donde se haga, pero esta no me puede alterar mi negociación (9). 
De acuerdo con las indagaciones realizadas con 23 entrevistados, se pudo determinar que la filosofía de negociación de los italianos se caracteriza por evitar el conflicto y la confrontación, mediante el uso de argumentos concretos, por eso quizá, manifiestan posiciones francas. "Para los italianos, los conflictos se ven como algo que se tiene que resolver, y no como un problema, son colaboradores, ven siempre el lado positivo y buscan soluciones (...) tratando de resolver el conflicto por el bien de todas las partes involucradas" (13).

Acostumbran ir preparados a la negociación, se proveen de suficientes evidencias basadas en criterios objetivos, apoyados en indagaciones propias, conocimientos y búsqueda de leyes y normas relativas a los asuntos a abordar; todo indica que van con el firme propósito de evitar cualquier tipo de riesgo que pudiera afectarles, ya que justamente tienen baja aceptación a la incertidumbre y a la derrota. La perspectiva temporal de esta cultura tiende hacia relaciones de largo plazo.

\subsection{Concepción de la contraparte}

En condiciones de alta confianza, la contraparte es apreciada, de manera particular cuando existen lazos de amistad o de buenas relaciones. Cuando los pares no han tenido contacto con la contraparte mexicana, despliegan amabilidad y cordialidad, demuestran su interés por las buenas relaciones. El negociador mexicano en general es serio, perfeccionista, profesional y con mucha formalidad; al hacer negociaciones dejan ver sus preferencias por llevar a cabo procesos negociadores con contrapartes que les inspiren la confianza necesaria, se sienten cómodos con contrapartes que les brindan amistad. "Los Trinitarios son cerrados, ya que es un esquema que requieren mucha información para generar confianza, les gusta ratificar las cosas, ir al lugar donde hicieron algo $y$ cerciorarse de que es verdad para poderlo aplicar. Ante todo cerciorarse y ver por ellos mismos ver si ha sido efectivo en otros países" (9).

Sus expectativas de relaciones de largo plazo son cotidianas. Aunque en general, tratan a la contraparte como colega, tienden a admirar a sus pares de países desarrollados y en ocasiones, cada vez menos, a minimizar a los negociadores provenientes de países de economías emergentes. "Los incidentes de la transacción fueron con el tiempo de entrega y el método de transporte, ya que la gente de Nigeria no confiaba en que al moverlo a otro país y enviarlo por barco llegaría a tiempo, por lo que fue necesario enviar evidencias que demostraran que era posible" (12).

En esta variable, la cultura italiana convive con su contraparte a través de un trato impersonal, pero cordial. No obstante, en ocasiones, los pares son percibidos como personas dignas de confianza y respeto, pero en otros casos, se les considera como un mero medio para el logro de sus propios intereses, se les minimiza, 
exige y presiona, especialmente si no se cuenta con las bases de confianza mutua. "Cuando los italianos negocian con otras culturas, se muestran desconfiados al comienzo, pero después cambian totalmente. A los italianos quizá les sorprenda lo cerradas que pueden ser otras culturas al momento de negociar... siempre expresan deseos de conocer a su contraparte, son muy elocuentes y comunicativos" (7).

Estas reacciones con tendencia por lo general a la autocomplacencia y soberbia de los negociadores italianos, no pasan inadvertidas para la contraparte, quien se queda con una percepción de desánimo y frustración. "La verdad es que me sentí utilizada (por los italianos). Intentaron utilizarme para llevar a cabo una serie de actividades que solo interesaban a ellos aprovechándose por un lado de mis desconocimientos sobre la materia y por el otro lado, el hecho de que ellos eran "los jefes" y yo el currito" (14).

Es conveniente destacar, como ya se ha hecho, que los italianos también son considerados negociadores muy educados y cordiales, con grandes deseos de apoyar a su contraparte y de buscar y proponer soluciones conjuntas que permitan el alcance de los objetivos mutuos. Diversos de los casos reportados para esta investigación, muestran a los italianos amistosos y creativos para resolver los conflictos que se han generado durante la negociación.

\subsection{Quiénes negocian}

El mexicano que negocia suele ser el de más alta jerarquía. En la actualidad, la decisión de quién negocia, está en función del tamaño de la empresa representada. "En el caso de Corea del Sur, había una reunión en Dubai; ellos y nosotros participamos y cada parte habló de lo que buscaba. Los dos estaban de acuerdo en que los directores de las dos empresas fueran a Dubai" (10).

Por ejemplo, si son entidades pequeñas o medianas, los negociadores serán los mismos dueños (llamados directores) y en caso de empresas grandes trasnacionales, cuentan con áreas de desarrollo de negocios o sus equivalentes, las cuales tienen el personal especializado, que además del conocimiento técnico, disponen de un esquema para tomar decisiones. Los negociadores mexicanos tienen puestos de gerentes o jefes que, a su vez, están apoyados por líderes de proyectos. "El gerente de la línea de Perfilaje de Pozos de Venezuela decide hacer una visita a OXY-Colombia para negociar en sus oficinas. Las personas que estarían presente serian: Gerente de Yacimientos de OXY-Colombia y Gerente Técnico. Gerente de la línea de productos de Perfilaje de Baker Hughes y Gerente Técnico (un servidor, mexicano)" (39). En general, el criterio de quién negocia se basa en la jerarquíaconocimiento.

En cuanto a los italianos, la investigación realizada no reúne datos suficientes 
que confirmen las actividades o las condiciones previas que se dan al interior de los grupos de negociadores italianos y las formas en que estos se eligen. Sin embargo, se puede inferir cierta tendencia jerárquica, es decir, el tomador de decisiones es el que negocia, o bien puede ser representado por sus colegas o subordinados que en general posean experiencia y conocimiento de los asuntos a acordar. Según el Instituto de Fomento Región de Murcia (2012) explica que las decisiones se toman de forma consensuada entre los miembros del equipo directivo, pero que es fundamental conocer qué imagen e influencia real tiene la persona que representa a la organización en la negociación.

La cultura italiana tiende a negociar con pares, por lo que la contraparte siempre deberá enviar representantes del mismo nivel jerárquico que sus colegas italianos, debido a que el involucramiento de su contraparte en el mismo rango es también un acto de formalidad. Asimismo, se aprecia cierto grado de profesionalización de los negociadores y respeto a la jerarquía. "Al ver que el proceso anterior en el nivel de gerentes de proyecto no rindió frutos, se decidió hacer una negociación de alto nivel entre las compañías integrantes del asocio... por parte de los italianos negociaría uno de los Vicepresidentes involucrados con los Proyectos en el exterior..." (17).

En consecuencia, se percibe un respeto a la constitución jerárquica que influye de manera singular en el cierre de las negociaciones. Por lo tanto, la toma de decisiones se lleva a cabo por esferas de poder, donde los niveles altos son preponderantes. Cuando el negociador actúa de manera independiente o goza de empoderamiento, quizá no acuda ante alguien más para decidir, pero lo más seguro es que asistirá a las negociaciones con un alto grado de preparación (Camacho, 2010).

\subsection{Preparación de la negociación}

Uno de los componentes fundamentales para lograr los propósitos y objetivos de las negociaciones con resultados óptimos para las organizaciones representadas por negociadores profesionales, sin duda, es la preparación que conlleva a la planeación y a la delimitación de la agenda de la negociación. La preparación de la negociación también incluye las prenegociaciones y las primeras aproximaciones entre las partes. Esto quiere decir que la preparación de la negociación es una actividad, por demás, significativa en el previo a un resultado óptimo del proceso.

El mexicano se prepara muy bien antes de iniciar una negociación formal, a través de recorridos de cortesía a empresas con quienes negocia, con el fin de conocer su contraparte; busca información que muestre evidencias de lo que él va ofrecer y lo que ofrecerá su contraparte, como el análisis de costos (acuerdos de precios), 
calidad de los productos, el fundamento técnico de trabajos o servicios, el idioma con el que se realizará la negociación. "Primero ellos nos contactaron por medio de nuestro proveedor principal AVEVA, nos contactó un ingeniero de Alemania de la compañía del escáner, todo en el idioma inglés. Investigamos en Internet su página a que se dedicaban y también con las referencias de AVEVA sobre otras países como Brasil que habían adquirido el hardware" (5).

Cuando el mexicano no posee el conocimiento del idioma, suele buscar intérpretes o personal calificado que lo pueda asesorar en la gran tarea de la consumación de los negocios; está muy interesado en establecer cláusulas de contratos, y no solo confía de palabra sino que también busca asegurarse con la firma de contratos, comprometiéndose él y su contraparte negociadora. "Las partes se hablaron por teléfono y el vendedor chino invito a 1 comprador mexicano, a que los visitara a su planta, y así podría ver la calidad y diversidad de los productos. El comprador mexicano fue al viaje de negocios con su hermano, para que tuviera más puntos de vista y el traductor de español a mandarín, y viceversa. El vendedor chino se preparó para ser cordial, amable y servicial, su objetivo era conocer mejor al comprador mexicano y venderle sus productos" (38).

Para los negociadores mexicanos la preparación de la negociación es, sin duda, una de las principales fases del proceso, ya que en esta parte los pares se proveen de información simétrica y asimétrica, que en ciertos casos puede ser motivo de intercambio entre las partes.

Esta etapa de la negociación es relevante para la cultura italiana, ya que la mayoría de las experiencias de los entrevistados, los refieren como negociadores muy formales. Bajo estas condiciones, es posible que se generen negociaciones informales, pero solo como un precedente de las negociaciones formales que se realicen. Se observa en los resultados obtenidos de las entrevistas, que los procesos previos a las negociaciones en la mesa son consensuados por los italianos en el nivel interno y con la contraparte, sobre todo para fines de establecer los asuntos que integrarán la agenda. "Primero, la parte que quiere lanzar el producto (España) explica todo su proyecto (a la contraparte italiana) a partir de alli se ve si técnicamente se puede llevar a cabo y si se puede, se empieza a negociar precio, plazo de entrega (20)".

Los italianos parecen ser muy cuidadosos con las prenegociaciones, ya que son una de las principales fuentes de información de las que se proveen para estructurar sus argumentos.

\subsection{Apertura de la negociación}

El mexicano, en general, se abastece de información estratégica antes de comenzar las negociaciones in situ, por lo que sus aperturas están basadas en criterios objetivos relacionados con los servicios o productos a negociar, y al establecimiento de 
precios; tiende al regateo porque es una forma de sentir que "ganan"; sin embargo, se adaptan con facilidad a las culturas no regateadoras como los europeos. Esto se debe a que invierte tiempo y recursos en la preparación de la negociación, investiga a su contraparte para conocer sus fortalezas y debilidades, y sobre estas últimas basa sus propuestas. Continuamente compara precios con otros proveedores y suele escuchar y analizar con atención la oferta de la contraparte.

El proceso fue en términos muy cordiales, cada quien estaba cierto que si bien era importante y conveniente para ambas partes continuar nuestra relación comercial, (se) tenían opciones para seguir logrando sus proyectos de negocios., por lo que al inicio de la negociación, fue una explicación y/o justificación para cada uno de los puntos solicitados para el posible acuerdo, la idea fue evaluar y separar aquellos que había confidencia o acuerdo para ser cumplidos y analizar a detalle los otros que no podían ser aceptados o que requerían un compromiso adicional o diferente. El proceso lo inició la empresa americana y poco a poco fuimos concluyendo en acuerdos satisfactorios, metas, y compromisos concretos para ambas partes (45).

La apertura en las negociaciones con italianos da muestra y llama mucho la atención lo bien preparados que van, pues sus escenarios están suficientemente fundamentados y hacen uso de información asimétrica. Las posiciones se presentan muy claramente desde la demanda inicial y en la mayoría de los casos reportados, parecen no moverse tan fácilmente. Las demandas son muy puntuales y se manifiestan desde el principio del proceso. "Fue una forma tradicional de negociación (con el italiano) con imposiciones y rechazo a las normas,(con) una postura muy firme en sus puntos de vista, sin opción a negociación...; se caracterizaba por ser muy frío y calculador en sus decisiones y su actuación; daba la impresión de estar programado hasta el mínimo detalle"(14).

En suma, el inicio de las negociaciones con italianos denota grandes dosis de datos concretos y con un enfoque de convencimiento a la contraparte, basado en una preparación previa que le permite argumentar con evidencias y generar así la confianza en el otro, y con ello el logro de sus propios objetivos.

\section{CONCLUSIONES}

En esta aproximación comparativa entre las formas de negociar de los mexicanos y de los italianos, se localizan datos que orientan el conocimiento de cómo negocian estas culturas y las prioridades que cada una de ellas toma en cuenta al llevar a cabo procesos de negociación con pares de otros países. Es elemental enfatizar que la información sobre las variables muestra la tendencias en el perfil negociador mexicano e italiano, respectivamente. Estos resultados deben ser confirmados con hallazgos de otras investigaciones sobre el tema. 
Concerniente a las variables revisadas en este artículo, la filosofía de negociación es un componente de lo que Hall (1989) denomina culturas de alto y bajo contexto; pero también es parte de la propuesta de Hofstede (1982) en cuanto a formas de expresión, incertidumbre y plazos. Asimismo se percibe la relación con las dimensiones propuestas por Trompenaars y Hampden-Turner (1997) en términos de emotividad y al binomio específico-difuso, inherente al nivel de tolerancia a la incertidumbre. Los hallazgos muestran que los mexicanos son una cultura de alto contexto; se caracterizan por llevar a cabo un proceso de distribución de valor, particularmente cuando no tienen confianza con la contraparte; su forma de expresión es policrónica y en ocasiones ambigua, sin comprometerse demasiado. Esta cultura negociadora prefiere no asumir riesgos, sin embargo, confían en las obligaciones que asume su contraparte. En cuanto al manejo de las emociones, suelen ser fraternos, hospitalarios, y tienden hacia la búsqueda de amistad para sentirse más cómodos y presumir sus buenas relaciones. Su perspectiva temporal denota una inclinación hacia el largo plazo.

Los italianos por su parte, evitan el conflicto y la confrontación mediante el uso de argumentos concretos y posiciones francas. Acostumbran ir preparados a la negociación, se proveen de suficientes evidencias basadas en criterios objetivos, por lo que sus formas de expresión son monocrónicas, apoyados en indagaciones propias, conocimientos y búsqueda de leyes y normas relativas a los asuntos a abordar; tienen baja aceptación a la incertidumbre y a la derrota. La perspectiva temporal de esta cultura tiende hacia relaciones de largo plazo. Es decir, se trata de una cultura de bajo contexto, aunque parecen otorgar valor, y según sea la contraparte, también lo distribuyen. Acostumbran ser considerados y atentos con sus pares.

En la concepción de la contraparte, la dimensión más destacada es la emotividad y formas de expresarse. Cuando los mexicanos se encuentran en condiciones de alta confianza, previos lazos de amistad o de buenas relaciones, dan un trato preferencial a la contraparte. No obstante, cuando aún no les conocen, pero aprecian un escenario promisorio, despliegan amabilidad y cordialidad, demuestran su interés por las buenas relaciones. El negociador mexicano en general es serio, perfeccionista, profesional y con mucha formalidad al hacer negociaciones. Se sienten cómodos con contrapartes que les brindan aprecio. Aunque en general tratan a la contraparte como colegas, tienden a admirar a sus pares de países desarrollados $y$, en ocasiones, cada vez menos, a minimizar a los negociadores provenientes de países de economías emergentes.

En esta variable, la cultura italiana convive con su contraparte a través de un trato impersonal, pero cordial. No obstante, en algunos casos, los pares son percibidos 
como personas dignas de confianza y respeto, pero en otros, se les minimiza, exige y presiona, especialmente si no se cuenta con las bases de confianza mutua. Estas reacciones no pasan inadvertidas para la contraparte, quien se queda con una percepción de desánimo y frustración. Sin embargo, es conveniente destacar que, en general, los italianos son considerados negociadores muy educados y cordiales, con grandes deseos de apoyar a su contraparte y de buscar y proponer soluciones conjuntas que permitan el alcance de los objetivos mutuos. Diversos de los casos reportados para esta investigación, muestran a los italianos amistosos y creativos para resolver los conflictos que se han generado durante la negociación.

Quiénes negocian es una variable que descansa en las dimensiones de distancia de poder (Hofstede) y logro o adscripción (Trompenaars y Hampden-Turner). El mexicano que negocia suele ser el de más alta jerarquía; del mismo modo influye el tamaño y el grado de madurez de la empresa. En cuanto a la cultura italiana aunque la información recabada no es contundente, se aprecia que tienden a negociar con pares, por razones de rango y formalidad. El tomador de decisiones es el que negocia, o bien puede ser representado por sus colegas o subordinados que posean experiencia y conocimiento de los asuntos a acordar. Según el Instituto de Fomento Región de Murcia (2012) explica que las decisiones se toman de forma consensuada entre los miembros del equipo directivo, pero es fundamental conocer qué imagen e influencia real tiene la persona que representa a la organización en la negociación.

Con respecto a la preparación, las principales propuestas involucradas son las de universalismo-particularismo y control interno o externo (Trompenaars y Hampden-Turner). El mexicano se prepara muy bien antes de iniciar una negociación formal, busca información que muestre evidencias de lo que él va ofrecer y lo que ofrecerá su contraparte, como el análisis de costos (acuerdos de precios), calidad de los productos, el fundamento técnico de trabajos o servicios, el idioma en el que se realizará la negociación. En la actualidad, este negociador se interesa en el establecimiento de cláusulas de contratos, y busca asegurarse de la firma respectiva, comprometiéndose él y su contraparte negociadora. Dada la formalidad de la cultura italiana, prenegocian solo como un precedente de las negociaciones formales que se realicen. De acuerdo con los resultados de la investigación, los procesos previos a las negociaciones en la mesa, son consensuadas por los italianos en el nivel interno y con la contraparte, sobre todo para fines de establecer los asuntos que integrarán la agenda.

La apertura está centrada en las formas de expresarse y la masculinidadfemineidad (Hofstede), así como el control interno-externo (Trompenaars y Ham- 
pden-Turner). El mexicano, comúnmente, se abastece de información estratégica antes de iniciar las negociaciones in situ, por lo que sus aperturas están basadas en criterios objetivos afines con los servicios o productos a negociar; se adaptan con facilidad a las culturas no regateadoras como los europeos. Esto es debido a que invierten tiempo y recursos en la preparación de la negociación, investigan a su contraparte para conocer sus fortalezas y debilidades, y sobre estas últimas basan sus propuestas. Los negociadores italianos van muy preparados en sus argumentos y criterios objetivos que darán a conocer en la apertura, sus alternativas parecen estar suficientemente fundamentadas y hacen uso de información asimétrica. Las posiciones se presentan muy claras desde la demanda inicial y en la generalidad de los casos reportados, parecen no cambiar de orientación fácilmente. Las demandas son muy puntuales y se dan a conocer desde el principio del proceso.

En suma, las culturas negociadoras de México e Italia, aunque en ubicaciones geográficas distantes, no son opuestas, sino complementarias. Se puede decir que los negociadores de estos países, cuando llevan a cabo procesos de esta especialidad, es muy probable que logren acuerdos óptimos; quizá un área de oportunidad donde convergen negativamente es en la expresión de emociones, aunque los mexicanos lo hacen de manera natural, los italianos lo manifiestan como una forma de poder y de imposición, es decir, emociones administradas.

Finalmente, es relevante mencionar que los procesos de negociaciones interculturales pueden ser más provechosos cuando se conoce el contexto cultural y el perfil negociador de la contraparte, lo cual proveerá un marco de comprensión, protocolo y alcance de objetivos mutuos y coadyuvará a la optimización de recursos organizacionales, tiempo y alcances de largo plazo.

\section{BIBLIOGRAFÍA}

Ambitalia (s.f.). Economía italiana. Consultado el 3 de enero de 2014.

Béjar, R. (2007). El mexicano: aspectos culturales y psicosociales. Séptima edición corregida y aumentada: 2007. México: UNAM

Calderón, E. et al (2010) Compiladora. Manual de negociaciones interculturales. El Salvador: Universidad de El Salvador.

Cerutti, C. (2006). Sulle differenze interculturali nelle trattative commerciali tra tedeschi e italiani. En: Economia aziendale 2000, N. 2/2006, 17 pp.

Camacho, M. (2010). Negociaciones italianas: un proceso intercultural, pp. 118-142. En: Ortiz, Rodríguez y Contreras Soto (Coordinadores). Interculturales. Edición electrónica gratuita.

CAMCIG (s/f). Perfil Italia. Consultado el 5 de febrero de 2013. 
Culturas negociadoras en México e Italia: una aproximación comparativa

Chamoun-Nicolas, H. (2005). Negociar con mexicanos es negociar con múltiples culturas.

Díaz-Guerrero, R. (1975). Psychology of the Mexican. Culture and personality. USA: The Texan pan American series.

Datosmacro.com (s/f). Población Italia. Recuperado el 4 de agosto de 2014 de: //www.datosmacro. com/demografia/población

García, E. (2005). Estilos de negociación vinculados a marcos culturales. Universidad de Vigo. Información interna. 11 pp.

Hendon, D. y Hendon R. (1999). Primera edición en español. Cómo negociar en cualquier parte del mundo. México: Limusa.

Hernández, R. Fernández, C. Baptista, P. (2010). Metodología de la investigación. (5. ․ Ed.). México: McGraw Hill.

Hofstede, G. (2011). Dimensionalizing Cultures: The Hofstede Model in Context. En: International Association for Cross-Cultural Psychology, Unidad 2, artículo 8, 26 pp.

Hofstede Centre The (s/f). Cultural Insights. Recuperado el 3 de agosto de 2013 de http://geert-hofstede.com/

Hofstede site. Dimensions of national Cultures. Disponible en http://geerthofstede.nl/dimensions-of-national-cultures

Horst, P. y Colonel, L. (2007). Cross-Cultural Negotiations. Air War College y Air University, USA, $37 \mathrm{pp}$.

INECC (2009). Actividades económicas. Instituto Nacional de Ecología y Cambio Climático, México. Consultado el 5 de febrero de 2013.

INEGI (s.f.). Territorio de México. Cuéntame... de México, Instituto Nacional de Estadística y Geográfica. Consultado el 7 de enero de 2014

Magallón, M. (2007). Samuel Ramos y su idea de cultura en México. Temas de Ciencia y Tecnología Vol. 11 Número 33 septiembre-diciembre 2007. págs. 13-22.

Martínez, C., Corredor, A. y Herazo, G. (2006). Negocios internacionales estrategias globales. Bogotá, Colombia: Universidad Santo Tomás.

OEI (s.f.). Perfil actual de México. Organización de Estados Iberoamericanos para la Educación, la Ciencia y la Cultura. Consultado el 17 de marzo de 2013.

Ogliastri, E. (1999). Una introducción a la negociación internacional. La cultura latinoamericana frente a la angloamericana, japonesa, francesa y del Medio Oriente. Universidad de los Andes. Serie Empresa, economía y sociedad. Monografía 40. Bogotá, Colombia.

Ogliastri, E. (1998). El estilo negociador de los latinoamericanos. Una investigación cualitativa. Revista de la Universidad de los Andes N. 41 enero-marzo, 198. págs. 70-83.

Ogliastri, E. Página personal disponible en http://enriqueogliastri.com/informes-investigacionresearch-reports/ 
Pariente F. J.L. (2003). Cultura y Administración intercultural. En: Administración y Organizaciones, Vol. 3, N. ${ }^{\circ}$ 6, México, julio, pp. 95-108.

Rosen, R. (2000). Éxito global y estrategia local. Buenos Aires: Vergara Business.

Secretaría de Economía (s/f). Información Sectorial. Secretaría Economía, SINEM, México.

SGM (s/f). Datos económicos Italia. Secretaría Economía, SINEM, México.

Trompenaars, F. E Hampden-Turner, Ch. (1997). Riding the Waves of Culture. London: Nicholas Brealey Publishing.

UNESCO (2001). Patrimonio mondiale. CNI UNESCO.

Unión Europea (s.f.).Italia. Consultado el 6 de mayo de 2013. 